\title{
Relatively small counterexamples to Hedetniemi's conjecture
}

\author{
Xuding Zhu*
}

June 30, 2020

\begin{abstract}
Hedetniemi conjectured in 1966 that $\chi(G \times H)=\min \{\chi(G), \chi(H)\}$ for all graphs $G$ and $H$. Here $G \times H$ is the graph with vertex set $V(G) \times V(H)$ defined by putting $(x, y)$ and $\left(x^{\prime}, y^{\prime}\right)$ adjacent if and only if $x x^{\prime} \in E(G)$ and $y y^{\prime} \in E(H)$. This conjecture received a lot of attention in the past half century. Recently, Shitov refuted this conjecture. Let $p$ be the minimum number of vertices in a graph of odd girth 7 and fractional chromatic number greater than $3+4 /(p-1)$. Shitov's proof shows that Hedetniemi's conjecture fails for some graphs with chromatic number about $p^{3} 3^{p}$ and with about $\left(p^{3} 3^{p}\right)^{p^{4} 3^{p-1}}$ vertices. In this paper, we show that the conjecture fails already for some graphs $G$ and $H$ with chromatic number $3\left\lceil\frac{p+1}{2}\right\rceil$ and with $p\lceil(p-1) / 2\rceil$ and $3\left\lceil\frac{p+1}{2}\right\rceil(p+1)-p$ vertices, respectively. The currently known upper bound for $p$ is 83 . Thus Hedetniemi's conjecture fails for some graphs $G$ and $H$ with chromatic number 126, and with 3, 403 and 10,501 vertices, respectively.
\end{abstract}

\section{Introduction}

The product $G \times H$ of graphs $G$ and $H$ has vertex set $V(G) \times V(H)$ and has $(x, y)$ adjacent to $\left(x^{\prime}, y^{\prime}\right)$ if and only if $x x^{\prime} \in E(G)$ and $y y^{\prime} \in E(H)$. Many names for this product are used in the literature, including the categorical product, the tensor product and the direct product. It is the most important product in this note. We just call it the product. We may write $x \sim y$ (in $G$ ) to denote $x y \in E(G)$.

A proper colouring $\phi$ of $G$ induces a proper colouring $\Phi$ of $G \times H$ defined as $\Phi(x, y)=$ $\phi(x)$. So $\chi(G \times H) \leq \chi(G)$. Symmetrically, we also have $\chi(G \times H) \leq \chi(H)$. Therefore $\chi(G \times H) \leq \min \{\chi(G), \chi(H)\}$. In 1966, Hedetniemi conjectured in 8 ] that equality always holds in the above inequality.

*Zhejiang Normal University, email:xdzhu@zjnu.edu.cn. Grant Numbers: NSFC 11971438 and 111 project of Ministry of Education of China. 
Conjecture 1 (Hedetniemi's conjecture) For any positive integer $c$, if $G \times H$ is $c$ colourable, then at least one of $G$ and $H$ is c-colourable.

This conjecture received a lot of attention in the past half century (see [1, 9, 12, 14, 22, 23]). Some special cases are confirmed. In particular, it was proved by El-Zahar and Sauer [1] that Hedetniemi's conjecture holds for $c=3$ (where for $c \leq 2$, the conjecture holds trivially). Also, it was proved in [23] that a fractional version of Hedetniemi's conjecture is true, i.e., for any graphs $G$ and $H, \chi_{f}(G \times H)=\min \left\{\chi_{f}(G), \chi_{f}(H)\right\}$.

Recently, Shitov refuted Hedetniemi's conjecture [13. He proved that Hedetniemi's conjecture fails for sufficiently large $c$. Let $p$ be the minimum number of vertices in a graph $G$ of odd girth 7 and fractional chromatic number greater than $3+4 /(p-1)$. Shitov's proof shows that Hedetniemi's conjecture fails for some $c$ about $3^{p} p^{3}$ and for graphs with about $c^{p^{3} 2^{p-1}}$ vertices. The current known upper bound for $p$ is 83 [17]. Thus Shitov's result shows that Hedetniemi's conjecture fails for some $c$ that is about $3^{95}$ and for graphs with about $\left(3^{95}\right)^{3^{99}}$ vertices.

On the other hand, we do not know if Hedetniemi's conjecture holds for any integer $c \geq 4$. A natural question is whether Hedetniemi's conjecture fails for relatively small $c$.

This paper shows that Hedetniemi's conjecture fails for some graphs $G$ and $H$ with chromatic number $3\lceil(p+1) / 2\rceil$ and with $p\lceil(p-1) / 2\rceil$ and

Using the upper bound $p \leq 83$, we conclude that Hedetniemi's conjecture fails for $c=125$ (and hence $G$ and $H$ can be assumed to have chromatic number 126). The number of vertices in $G$ and $H$ are 3,403 and 10,501, respectively.

\section{$2 \quad$ Exponential graph}

One of the standard tools used in the study of Hedetniemi's conjecture is the concept of exponential graphs. Let $c$ be a positive integer. We denote by $[c]$ the set $\{1,2, \ldots, c\}$, and for integers $c \leq d$, let $[c, d]=\{c, c+1, \ldots, d\}$. For a graph $G$, the exponential graph $K_{c}^{G}$ has vertex set

$$
\{f: f \text { is a mapping from } V(G) \text { to }[c]\},
$$

with $f g \in E\left(K_{c}^{G}\right)$ if and only if for any edge $x y \in E(G), f(x) \neq g(y)$. In particular, $f \sim f$ is a loop in $K_{c}^{G}$ if and only if $f$ is a proper $c$-colouring of $G$. So if $\chi(G)>c$, then $K_{c}^{G}$ has no loop.

For two graphs $G$ and $H$, a homomorphism from $G$ to $H$ is a mapping $\phi: V(G) \rightarrow$ $V(H)$ that preserves edges, i.e., for every edge $x y$ of $G, \phi(x) \phi(y)$ is an edge of $H$. We say $G$ is homomorphic to $H$, and write $G \rightarrow H$, if there is a homomorphism from $G$ to $H$. The "homomorphic" relation " $\rightarrow$ " is a quasi-order. It is reflexive and transitive: if $G \rightarrow H$ and $H \rightarrow Q$ then $G \rightarrow Q$. The composition $\psi \circ \phi$ of a homomorphism $\phi$ from $G$ to $H$ and a homomorphism $\psi$ from $H$ to $Q$ is a homomorphism from $G$ to $Q$.

Note that a homomorphism from a graph $G$ to $K_{c}$ is equivalent to a proper $c$-colouring of $G$. Thus if $G \rightarrow H$, then $\chi(G) \leq \chi(H)$. The following result was proved in [1]. For the completeness of this paper, we include a short proof. 
Lemma 2 ([1]) For any graph $H, \chi(G \times H) \leq c$ if and only if $H$ is homomorphic to $K_{c}^{G}$.

Proof. The maps $\Psi: V(G) \times V(H) \rightarrow[c]$ naturally correspond to the maps $f: V(H) \rightarrow$ $V\left(K_{c}^{G}\right)$ via $f(u)(v)=\Psi(v, u)$. This is a $1-1$ correspondence where proper colorings correspond to exactly the homomorphisms, i.e., $\Psi$ is a proper colouring if and only if $f$ is a homomorphism from $F$ to $K_{c}^{G}$.

So $K_{c}^{G}$ is the largest graph $H$ in the order of homomorphism with the property that $\chi(G \times H) \leq c$. Thus Conjecture 1 is equivalent to the following statement:

(*) For any positive integer $c$, if $\chi(G)>c$, then $\chi\left(K_{c}^{G}\right) \leq c$.

\section{Construction of a counterexample}

The lexicographic product $G[H]$ of two graphs $G$ and $H$ is the graph obtained from $G$ by replacing each vertex of $G$ by a copy of $H$. Thus the graph $G\left[K_{q}\right]$ has vertex set $\{(x, i): x \in V(G), i \in[q]\}$, where $(x, i)$ and $(y, j)$ are adjacent if and only if either $x y \in E(G)$ or $x=y$ and $i \neq j$. The fractional chromatic number $\chi_{f}(G)$ of $G$ is defined as

$$
\chi_{f}(G)=\inf \left\{\chi\left(G\left[K_{q}\right]\right) / q: q=1,2, \ldots\right\} .
$$

The odd girth of $G$ is the length of a shortest odd cycle in $G$.

Let $p$ be the minimum order of a graph of odd girth 7 and with fractional chromatic number greater than $3+\frac{4}{p-1}$. Let $F$ be such a graph. The existence of $F$ is guaranteed by a classical result of Erdös [2] that there are graphs $F$ with $\operatorname{girth}(F) \geq g$ and $\chi_{f}(F) \geq r$ for any $g, r$ (usually it is stated as $\chi(F) \geq r$, but the proof actually shows that $\left.\chi_{f}(F) \geq r\right)$. In Section 4, we shall discuss more on the order $p$ of $F$. We assume $V(F)=\left\{v_{1}, v_{2}, \ldots, v_{p}\right\}$.

Let $q \geq(p-1) / 2$ be an integer and $c=3 q+2$. Let $G=F\left[K_{q}\right]$. Let $H$ be the graph that consists of

(1) vertices $g_{i}$ for $i \in[c]$ forming a clique;

(2) a vertex $\phi$ adjacent to $g_{i}$ for $i>p$;

(3) for each $i \in[p]$, for each $t \in[q+2,3 q+2]$, a vertex $\mu_{i, t}$, where for each $i \in[p]$, the set $\left\{\mu_{i, t}: t \in[q+2,3 q+2]\right\} \cup\left\{g_{i}\right\}$ forms a clique, and the vertex $\mu_{i, t}$ is further adjacent to $g_{j}$ with $j>2 q+1, j \neq t$;

(4) for each $i \in[p]$ and $t \in[2 q+2,3 q+2]$, a vertex $\theta_{i, t}$ adjacent to $\phi$ and $\mu_{i, t}$ and to each $g_{j}$ with $j \notin\{i, t\}$.

Theorem $3 \chi(G), \chi(H)>c$ and $\chi(G \times H) \leq c$. 


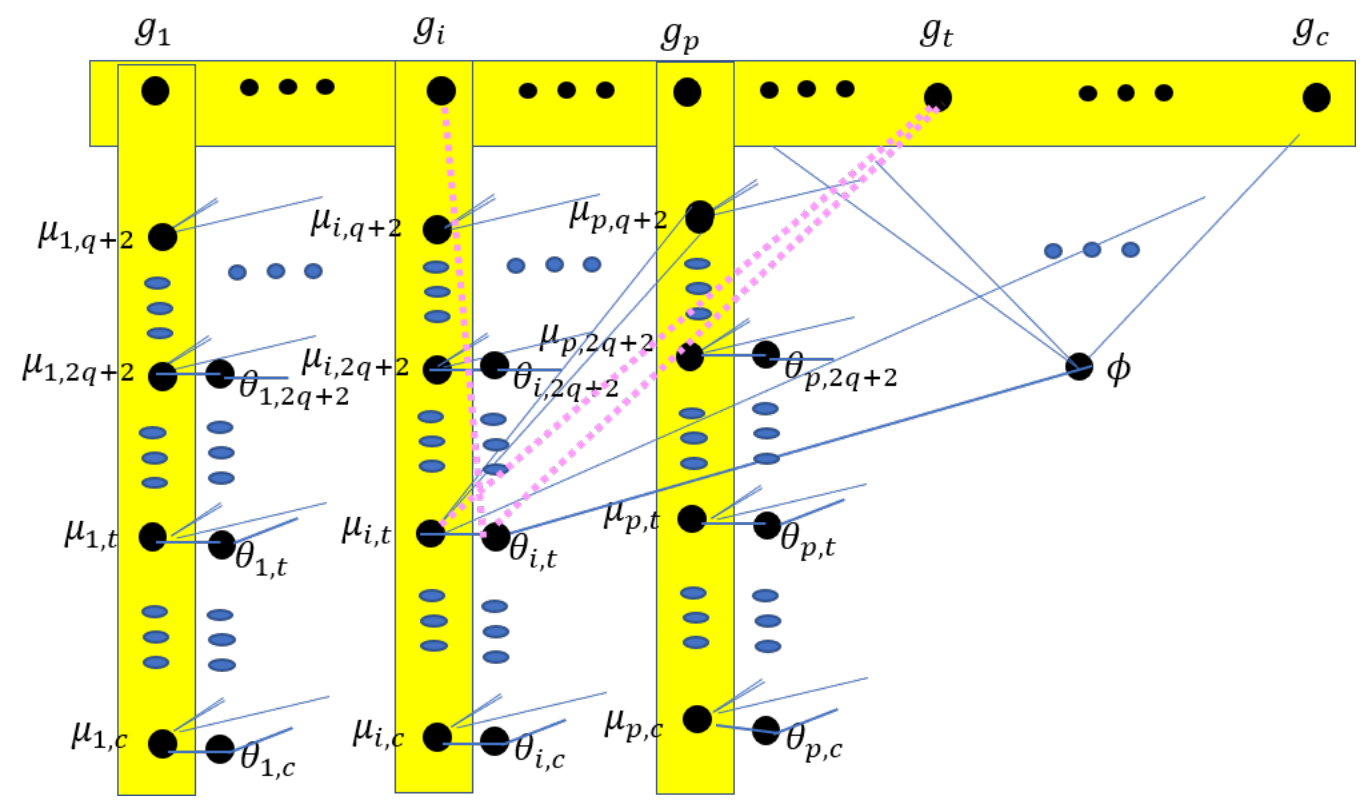

Figure 1: The graph $H$, where each shaded rectangle is a clique

Proof. It follows from the definition of fractional chromatic number that

$$
\chi(G)=\chi\left(F\left[K_{q}\right]\right) \geq q \chi_{f}(F)>3 q+2=c .
$$

Next we show that $\chi(H)>c$. Assume to the contrary that $\chi(H) \leq c$ and $\Psi$ is a $c$-colouring of $H$. We may assume that $\Psi\left(g_{i}\right)=i$.

As $\phi$ is adjacent to $g_{j}$ for $j>p$, we conclude that $\Psi(\phi)=i^{\star}$ for some $i^{\star} \leq p$.

As the set $\left\{\mu_{i^{*}, t}: t \in[q+2,3 q+2]\right\} \cup\left\{g_{i^{*}}\right\}$ forms a clique of $2 q+2$ vertices and $\Psi\left(g_{i^{*}}\right)=i^{*} \leq 2 q+1$, there exists $t^{*} \in[2 q+2,3 q+2]$ such that $\Psi\left(\mu_{i^{*}, t^{*}}\right) \geq 2 q+2$. As $\mu_{i^{*}, t^{*}}$ is adjacent to all $g_{j}$ with $j \in[2 q+2,3 q+2]-\left\{t^{*}\right\}$, we conclude that $\Psi\left(u_{i^{*}, t^{*}}\right)=t^{*} \geq 2 q+2$.

The vertex $\theta_{i^{*}, t^{*}}$ is adjacent to each $g_{j}$ with $j \notin\left\{i^{*}, t^{*}\right\}$. Hence $\Psi\left(\theta_{i^{*}, t^{*}}\right) \in\left\{i^{*}, t^{*}\right\}$. But $\theta_{i^{\star}, t^{*}}$ is adjacent to $\phi$, which is coloured by $i^{*}$, and adjacent to $\mu_{i^{*}, t^{*}}$, which is coloured by $t^{*}$. This is a contradiction, and thus $\chi(H)>c$.

It remains to show that $\chi(G \times H) \leq c$. By Lemma 2, it suffices to show that $H$ is homomorphic to $K_{c}^{G}$. We shall prove that $H$ is actually a subgraph of $K_{c}^{G}$. For this purpose, we simply define each vertex $y$ of $H$ as a mapping $y: V(G) \rightarrow[c]$ and show that if $y y^{\prime} \in E(H)$, then $y y^{\prime} \in E\left(K_{c}^{G}\right)$, i.e., for any edge $x x^{\prime} \in E(G), y(x) \neq y^{\prime}\left(x^{\prime}\right)$. (This is equivalent to say that the mapping $\Phi: V(G \times H) \rightarrow[c]$ defined as $\Phi(x, y)=y(x)$ is a proper $c$-colouring of $G \times H)$.

Each vertex of $G$ is of the form $\left(v_{s}, j\right), s \in[p]$ and $j \in[q]$.

For $y \in V(H)$, let $y: V(G) \rightarrow[c]$ be defined as follows:

1. For $i \in[c]$ and $\left(v_{s}, j\right) \in V(G), g_{i}\left(v_{s}, j\right)=i$.

2. For $\left(v_{s}, j\right) \in V(G), \phi\left(v_{s}, j\right)=s$. 
3. For $i \in[p], t \in[q+2,3 q+2]$ and $\left(v_{s}, j\right) \in V(G)$,

$$
\mu_{i, t}\left(v_{s}, j\right)= \begin{cases}j+\delta_{j \geq i}, & \text { if } d_{F}\left(v_{s}, v_{i}\right)=0 \text { or } 2, \\ q+j+\delta_{q+j \geq i}, & \text { if } d_{F}\left(v_{s}, v_{i}\right)=1, \\ t-\delta_{i \geq t}, & \text { if } d_{F}\left(v_{s}, v_{i}\right) \geq 3,\end{cases}
$$

where

$$
\delta_{j \geq i}= \begin{cases}1, & \text { if } j \geq i \\ 0, & \text { otherwise }\end{cases}
$$

4. For $i \in[p], t \in[2 q+2,3 q+2]$ and $\left(v_{s}, j\right) \in V(G)$,

$$
\theta_{i, t}\left(v_{s}, j\right)= \begin{cases}i, & \text { if } d_{F}\left(v_{s}, v_{i}\right) \geq 2 \\ t, & \text { if } d_{F}\left(v_{s}, v_{i}\right) \leq 1\end{cases}
$$

Now we show that if $y y^{\prime} \in E(H)$, then $y y^{\prime} \in E\left(K_{c}^{G}\right)$. For $y \in V(H)$, let

$$
\operatorname{Im}(y)=\{y(x): x \in V(G)\} .
$$

Recall that $y y^{\prime} \in E\left(K_{c}^{G}\right)$ if and only if for every edge $x x^{\prime} \in E(G), y(x) \neq y^{\prime}\left(x^{\prime}\right)$. So we have the following easy observation.

Observation 4 If $\operatorname{Im}(y) \cap \operatorname{Im}\left(y^{\prime}\right)=\varnothing$, then $y y^{\prime} \in E\left(K_{c}^{G}\right)$.

Observation 5 For $i \in[p], t \in[q+2,3 q+2]$, the following hold:

- If $d_{F}\left(v_{s}, v_{i}\right)=0$ or 2 , then $\mu_{i, t}\left(v_{s}, j\right)<i$ when $j<i$ and $\mu_{i, t}\left(v_{s}, j\right)>i$ when $j \geq i$.

- If $d_{F}\left(v_{s}, v_{i}\right)=1$, then $\mu_{i, t}\left(v_{s}, j\right)<i$ when $q+j<i$ and $\mu_{i, t}\left(v_{s}, j\right)>i$ when $q+j \geq i$.

- If $d_{F}\left(v_{s}, v_{i}\right) \geq 3$, then $\mu_{i, t}\left(v_{s}, j\right)<i$ when $t \leq i$ and $\mu_{i, t}\left(v_{s}, j\right)>i$ when $t>i$.

So $i \notin \operatorname{Im}\left(\mu_{i, t}\right)$ and hence

$$
\operatorname{Im}\left(\mu_{i, t}\right)=([2 q+1] \cup\{t\})-\{i\} .
$$

Moreover,

$$
\operatorname{Im}(\phi)=[p], \operatorname{Im}\left(\theta_{i, t}\right)=\{i, t\} .
$$

In the construction of $H$, the vertices and edges of $H$ are added in four steps. Now we show that $y y^{\prime} \in E(H)$ implies that $y y^{\prime} \in E\left(K_{c}^{G}\right)$ according to these four steps.

(1) For any $i \neq j, \operatorname{Im}\left(g_{i}\right) \cap \operatorname{Im}\left(g_{j}\right)=\{i\} \cap\{j\}=\varnothing$. So $\left\{g_{i}: i \in[c]\right\}$ induces a clique.

(2) For $j>p, \operatorname{Im}(\phi) \cap \operatorname{Im}\left(g_{j}\right)=\varnothing$. So $\phi \sim g_{j}$ in $K_{c}^{G}$. 
(3) For $i \in[p]$ and $t \in[q+2,3 q+2]$, for $j \in\{i\} \cup([2 q+2,3 q+2]-\{t\}), \operatorname{Im}\left(\mu_{i, t}\right) \cap \operatorname{Im}\left(g_{j}\right)=$ $\varnothing$. So $\mu_{i, t} \sim g_{j}$ in $K_{c}^{G}$.

Now we show that if $t \neq t^{\prime}$, then $\mu_{i, t} \sim \mu_{i, t^{\prime}}$ in $K_{c}^{G}$. Assume to the contrary that $\mu_{i, t} \psi$ $\mu_{i, t^{\prime}}$ in $K_{c}^{G}$. Then there exists $\left(v_{s}, j\right)\left(v_{s^{\prime}}, j^{\prime}\right) \in E(G)$ such that $\mu_{i, t}\left(v_{s}, j\right)=\mu_{i, t^{\prime}}\left(v_{s^{\prime}}, j^{\prime}\right)$. Let $\alpha=\mu_{i, t}\left(v_{s}, j\right)=\mu_{i, t^{\prime}}\left(v_{s^{\prime}}, j^{\prime}\right)$. Then

$$
\alpha \in\left\{j+\delta_{j \geq i}, q+j+\delta_{q+j \geq i}, t-\delta_{i \geq t}\right\} \cap\left\{j^{\prime}+\delta_{j^{\prime} \geq i}, q+j^{\prime}+\delta_{q+j^{\prime} \geq i}, t^{\prime}-\delta_{i \geq t^{\prime}}\right\} .
$$

For $a \in\left\{t, t^{\prime}\right\}$ and $b \in\left\{j, j^{\prime}\right\}$, since $a \geq q+2$ and $b \leq q$, we have $\delta_{i \geq a}+\delta_{b \geq i} \leq 1$. Hence $b+\delta_{b \geq i}<a-\delta_{i \geq a}$.

We consider two cases.

Case $1 j \neq j^{\prime}$.

In this case, $j+\delta_{j \geq i} \notin\left\{j^{\prime}+\delta_{j^{\prime} \geq i}, q+j^{\prime}+\delta_{q+j^{\prime} \geq i}, t^{\prime}-\delta_{i \geq t^{\prime}}\right\}$ and $j^{\prime}+\delta_{j^{\prime} \geq i} \notin\left\{j+\delta_{j \geq i}, q+j+\right.$ $\left.\delta_{q+j \geq i}, t-\delta_{i \geq t}\right\}$. So

$$
\alpha \in\left\{q+j+\delta_{q+j \geq i}, t-\delta_{i \geq t}\right\} \cap\left\{q+j^{\prime}+\delta_{q+j^{\prime} \geq i}, t^{\prime}-\delta_{i \geq t^{\prime}}\right\} .
$$

As $t-\delta_{i \geq t} \neq t^{\prime}-\delta_{i \geq t^{\prime}}$ and $q+j+\delta_{q+j \geq i} \neq q+j^{\prime}+\delta_{q+j^{\prime} \geq i}$, we may assume that $\alpha=q+j+\delta_{q+j \geq i}=$ $t^{\prime}-\delta_{i \geq t^{\prime}}$. This implies that $d_{F}\left(v_{i}, v_{s}\right)=1$ and $d_{F}\left(v_{s^{\prime}}, v_{i}\right) \geq 3$, in contrary to the assumption that $\left(v_{s}, j\right)\left(v_{s^{\prime}}, j^{\prime}\right) \in E(G)$.

Case $2 j=j^{\prime}$.

As $\left(v_{s}, j\right) \neq\left(v_{s^{\prime}}, j\right)$, we have $v_{s} \neq v_{s^{\prime}}, v_{s} v_{s^{\prime}} \in E(F)$, and $\alpha \in\left\{j+\delta_{j \geq i}, q+j+\delta_{q+j \geq i}\right\}$. If $\alpha=j+\delta_{j \geq i}$, then $d_{F}\left(v_{s}, v_{i}\right), d_{F}\left(v_{s^{\prime}}, v_{i}\right) \in\{0,2\}$. As $v_{s} v_{s^{\prime}} \in E(F)$, this implies that $F$ has a 3-cycle or a 5-cycle, contrary to the assumption that $F$ has odd girth 7 .

Assume $\alpha=q+j+\delta_{q+j \geq i}$. It is possible that $\alpha \in\left\{t-\delta_{i \geq t}, t^{\prime}-\delta_{i \geq t^{\prime}}\right\}$. As $t-\delta_{i \geq t} \neq t^{\prime}-\delta_{i \geq t^{\prime}}$, by symmetry, we assume that $q+j+\delta_{q+j \geq i} \neq t-\delta_{i \geq t}$. Then $\mu_{i, t}\left(v_{s}, j\right)$ is defined by the second line of the definition of $\mu_{i, t}$. Hence $d_{F}\left(v_{s}, v_{i}\right)=1$. As $v_{s} v_{s^{\prime}} \in E(F)$, we conclude that $d_{F}\left(v_{s^{\prime}}, v_{i}\right) \leq 2$. This implies that $\mu_{i, t}\left(v_{s^{\prime}}, j\right)$ is not defined by the third line of the definition of $\mu_{i, t}$, even though $\mu_{i, t}\left(v_{s^{\prime}}, j\right)$ possibly equals $t^{\prime}-\delta_{i \geq t^{\prime}}$. So $\mu_{i, t}\left(v_{s^{\prime}}, j\right)$ is also defined by the second line of the definition of $\mu_{i, t}$. Hence $d_{F}\left(v_{s^{\prime}}, v_{i}\right)=1$. Then $F$ has a 3 -cycle, again a contradiction.

(4) For $i \in[p]$ and $t \in[2 q+2,3 q+2]$, as $\operatorname{Im}\left(\theta_{i, t}\right)=\{i, t\}$, we know that $\theta_{i, t} \sim g_{j}$ in $K_{c}^{G}$ for $j \notin\{i, t\}$. It remains to show that $\theta_{i, t} \sim \mu_{i, t}$ and $\theta_{i, t} \sim \phi$ in $K_{c}^{G}$.

If $\theta_{i, t} \psi \mu_{i, t}$ in $K_{c}^{G}$, then there exists $\left(v_{s}, j\right)\left(v_{s^{\prime}}, j^{\prime}\right) \in E(G)$ such that $\theta_{i, t}\left(v_{s}, j\right)=$ $\mu_{i, t}\left(v_{s^{\prime}}, j^{\prime}\right) \in \operatorname{Im}\left(\theta_{i, t}\right) \cap \operatorname{Im}\left(\mu_{i, t}\right)=\{t\}$. But $\theta_{i, t}\left(v_{s}, j\right)=t$ implies that $d_{F}\left(v_{s}, v_{i}\right) \leq 1$, and $\mu_{i, t}\left(v_{s^{\prime}}, j^{\prime}\right)=t$ implies that $d_{F}\left(v_{s^{\prime}}, v_{i}\right) \geq 3$ (note that as $i \leq p \leq 2 q+1$ and $t \geq$ $2 q+2, \mu_{i, t}\left(v_{s^{\prime}}, j^{\prime}\right)=t-\delta_{i \geq t}=t$ when $\left.d_{F}\left(v_{s^{\prime}}, v_{i}\right) \geq 3\right)$, in contrary to the assumption that $\left(v_{s}, j\right)\left(v_{s^{\prime}}, j^{\prime}\right) \in E(G)$.

If $\theta_{i, t} \not \phi$ in $K_{c}^{G}$, then there exists $\left(v_{s}, j\right)\left(v_{s^{\prime}}, j^{\prime}\right) \in E(G)$ such that $\theta_{i, t}\left(v_{s}, j\right)=$ $\phi\left(v_{s^{\prime}}, j^{\prime}\right) \in \operatorname{Im}\left(\theta_{i, t}\right) \cap \operatorname{Im}(\phi)=\{i\}$. But $\theta_{i, t}\left(v_{s}, j\right)=i$ implies that $d_{F}\left(v_{s}, v_{i}\right) \geq 2$, and $\phi\left(v_{s^{\prime}}, j^{\prime}\right)=i$ implies that $s^{\prime}=i$, again in contrary to the assumption that $\left(v_{s}, j\right)\left(v_{s^{\prime}}, j^{\prime}\right) \in$ $E(G)$.

This completes the proof of Theorem 3 . 


\section{Some remarks and Questions}

(1) As observed in Section 3, the existence of a graph $F$ of odd girth 7, order $p$ and fractional chromatic number greater than $3+4 /(p-1)$ is guaranteed by a classical result of Erdös [2]. Let $p$ be the minimum order of an odd girth 7 graph $F$ with $\chi_{f}(F)>$ $3+4 /(p-1)$. The probabilistic proof in [2] can be used to derive an upper bound on $p$. However, that bound is very big. The first relatively small upper bound on $p$, which I learned from Anna Gujgiczer and Gábor Simonyi [4], is obtained by using the generalized Mycielski construction (cf. [5, 16]). Let $P_{r}$ be obtained from the path $v_{0} v_{1} \ldots v_{r}$ by adding a loop at vertex $v_{0}$. The generalized Mycielski graph $M_{r}(G)$ of $G$ is obtained from $G \times P_{r}$ by identifying all the vertices whose second coordinate is $v_{r}$. So $M_{1}(G)$ is obtained from $G$ by adding a universal vertex, and $M_{2}(G)$ is the Mycielski graph of $G$. Tardif [16] showed that for any graph $G$ and any positive integer $r$,

$$
\chi_{f}\left(M_{r}(G)\right)=\chi_{f}(G)+\frac{1}{\sum_{i=0}^{r-1}\left(\chi_{f}(G)-1\right)^{i}} .
$$

For an integer vector $\vec{r}=\left(r_{1}, r_{2}, \ldots, r_{d}\right)$, let $M_{\vec{r}}(G)=M_{r_{1}}\left(M_{r_{2}} \ldots\left(M_{r_{d}}(G) \ldots\right)\right)$. Let $G=M_{(3,3,3,3)}\left(C_{7}\right)$. Then $G$ has 607 vertices, odd girth 7 , and $\chi_{f}(G)>3.09$ according to the above formula.

After the preliminary version of this paper, I learned from Geoffrey Exoo and Jan Goedgebeur [3] that they found an odd girth 7 graph $G$ on 49 vertices with independence number 17, through a computer search. Then $M_{3}(G)$ is an odd girth 7 graph on 148 vertices with fractional chromatic number greater than 3.03. Later, Geoffrey Exoo found an odd girth 7 graph $G$ on 83 vertices and with independence number 27. This implies that $\chi_{f}(G)>3.074$. So $p \leq 83$. The exact value of $p$ remains an open problem.

Question 6 What is the minimum order $p$ of a graph $F$ of odd girth 7 and with $\chi_{f}(F)>$ $3+4 /(p-1)$ ? In general, what is the minimum order $n_{k, t}$ of a graph with odd girth at least $k$ and fractional chromatic number at least $t$ ?

With $c=3 q+2$ and $q=\left\lceil\frac{p-1}{2}\right\rceil$, the number of vertices in $H$ is

$$
3 q+2+1+p(2 q+1)+p(q+1)=3\left\lceil\frac{p+1}{2}\right\rceil(p+1)-p .
$$

The graph $G=F\left[K_{q}\right]$ has $p q$ vertices. For $p=83$, we have $q=41$ and $c=125$. The two graphs $G$ and $H$ in Theorem 3 have 3,403 and 10,501 vertices, respectively.

Exoo also found an odd girth 7 graph $G$ on 84 vertices and with independence number 27. Then $\chi_{f}(G) \geq 3+1 / 9$. In the proof of Theorem 3, $q$ can be any integer at least $(p-1) / 2$. Also instead of $c=3 q+2$, we may choose $c=3 q+3$ or $3 q+4$, provided that we can guarantee that $\chi(G) \geq \chi_{f}(F) q>c$. By choosing $q$ and $c$ appropriately, we can show that Hedetniemi's conjecture fails for all $c \geq 125$, except that for $c=127$ (if we choose $p=83, q=41, c=3 q+4$, then we cannot guarantee that $\chi(G)>c)$. 
(2) The Poljak-Rödl function is defined in [11]:

$$
f(n)=\min \{\chi(G \times H): \chi(G), \chi(H) \geq n\} .
$$

Hedetniemi's conjecture is equivalent to saying that $f(n)=n$ for all positive integer $n$. Shitov's Theorem says that for sufficiently large $n, f(n) \leq n-1$. Using Shitov's result, Tardif and Zhu [20] proved that $f(n) \leq n-(\log n)^{1 / 4-o(1)}$ for sufficiently large $n$. Tardif and Zhu asked in [20] if there is a positive constant $\epsilon$ such that $f(n) \leq(1-\epsilon) n$ for sufficiently large $n$. This question was answered in affirmative by He and Wigderson [7] with $\epsilon \approx 10^{-9}$. Recently, Zhu [24] proved that $\lim _{\sup } \ln _{n \rightarrow \infty} \frac{f(n)}{n} \leq \frac{1}{2}$.

On the other hand, the only known lower bound for $f(n)$ is that $f(n) \geq 4$ for $n \geq 4$. An intriguing question is whether $f(n)$ goes to infinity with $n$.

Question 7 ([11]) Is $f(n)$ bounded by a constant or $\lim _{n \rightarrow \infty} f(n)=\infty$ ?

It was proved in [11] that if $f(n)$ is bounded by a constant, then the smallest such constant is at most 16. This result is further improved in [10] (see also [22]) that the smallest such constant is at most 9. To gain insight to this problem, it is still interesting to study Hedetniemi's conjecture for small integers $c$.

The fractional version of Hedetniemi's conjecture was proved in [23]: For any two graphs $G$ and $H, \chi_{f}(G \times H)=\min \left\{\chi_{f}(G), \chi_{f}(H)\right\}$. Thus if $f(n)$ is bounded by 9 , and $G$ and $H$ are $n$-chromatic graphs with $\chi(G \times H) \leq 9$, then at least one of $G$ and $H$ has fractional chromatic number at most 9.

In [23], the following Poljak-Rödl type function was defined:

$$
\psi(n)=\min \left\{\chi(G \times H): \chi_{f}(G), \chi(H) \geq n\right\} .
$$

It follows from the definition that $f(n) \leq \psi(n)$. This author proposed a weaker version of Hedetniemi's conjecture in [23], which is equivalent to the statement that $\psi(n)=n$ for all positive integer $n$. However, Shitov's proof actually refutes this weaker version of Hedetniemi's conjecture. The same is true for the result in this paper, as the graph $G$ used in the proof of Theorem 3 has large fractional chromatic number.

The proof in [24] actually shows that

$$
\limsup _{n \rightarrow \infty} \frac{\psi(n)}{n} \leq \frac{1}{2}
$$

A natural question is the following:

Question 8 Is $\psi(n)$ bounded by a constant? If $\psi(n)$ is bounded by a constant, what could be the smallest such constant?

(3) The result of El-Zahar and Sauer that Hedetniemi's conjecture holds for $c=3$ is still the best result in the positive direction of Hedetniemi's conjecture. On the 
other hand, there are nice strengthenings of this result, in the setting of multiplicative graphs. We say a graph $Q$ is multiplicative if for any two graphs $G, H, G \nrightarrow Q$ and $H \nrightarrow Q$ implies that $G \times H \nrightarrow Q$. Hedetniemi's conjecture is equiavelnt to the statement that $K_{c}$ is multiplicative for any positive integer $c$. El-Zahar and Sauer proved that $K_{3}$ is multiplicative. Häggkvist, Hell, Miller and Neumann Lara [6] proved that odd cycles are multiplicative and Tardif [15] proved that circular cliques $K_{k / d}$ for $k / d<4$ are multiplicative, where $K_{k / d}$ has vertex set [k] with $i \sim j$ if and only if $d \leq|i-j| \leq k-d$. (So $K_{k / 1}=K_{k}$ and $K_{(2 k+1) / k}=C_{2 k+1}$ ). In 2017, Wrochna 21] extended greatly the family of known multiplicative graphs by showing that any graph without 4-cycles is multiplicative. This result is further improved in [19] where it is shown that graphs in which each edge lies in at most one 4-cycle are multiplicative, and also that third powers of graphs with girth at least 13 are multiplicative.

Acknowledgement I would like to thank Gábor Tardos for inspiring comments that led to further reduction on the chromatic number and the number of vertices of the involved graphs from an earlier version, and for many comments that improve the presentation of this paper. I also thank Eun-Kyung Cho, Geoffrey Exoo, Jan Goedgebeur, Anna Gujgiczer, Benjamin R. Moore, Yaroslav Shitov, Gábor Simonyi and Claude Tardif for valuable comments and discussions and for the efforts of finding and reducing the upper bounds for $p$ and keep me informed.

\section{References}

[1] M. El-Zahar, N.W. Sauer, The chromatic number of the product of two 4-chromatic graphs is 4, Combinatorica 5 (1985) 121-126.

[2] P. Erdős, Graph theory and probability, Canad. J. Math. 11 (1959), 3438.

[3] G. Exoo and J. Goedgebeur, personal communication, 2020.

[4] A. Gujgiczer and G. Simonyi, personal communication, 2020.

[5] A. Gyárfás, T. Jensen and M. M. Stiebitz, On graphs with strongly independent color classes, J. Graph Th. 46 (2004), 1-14.

[6] R. Häggkvist, P. Hell, J. Miller and V. Neumann Lara, On multiplicative graphs and the product conjecture, Combiantorica 8(1988), 71-81.

[7] X. He and Y. Wigderson, Hedetniemi's conjecture is asympototically false, Journal of Combinatorial Theory, Ser. B, 10.1016/j.jctb.2020.03.003.

[8] S. Hedetniemi, Homomorphisms of graphs and automata, Technical Report 0310544-T, University of Michigan, 1966.

[9] S. Klavžar, Coloring graph products - a survey, Discrete Math. 155 (1996) 135-145. 
[10] S. Poljak, Coloring digraphs by iterated antichains, Comment. Math. Univ. Carolin. 32(1991), 209-212.

[11] S. Poljak and V. Rödl, On the arc-chromatic number of a digraph, J. Combin. Theory Ser. B 31(1981), 339-350.

[12] N. Sauer, Hedetniemi's conjecture - a survey, Discrete Math. 229 (2001) 261-292.

[13] Y. Shitov, Counterexamples to Hedetniemi's Conjecture, Ann. of Math. (2) 190(2019), no. 2, 663667.

[14] C. Tardif, Hedetniemi's conjecture, 40 years later, Graph Theory Notes N. Y. 54 (2008) 46-57.

[15] C. Tardif, Multiplicative graphs and semi-lattice endomorphisms in the category of graphs, Journal of Combinatorial Theory Ser. B 95 (2005), 338-345.

[16] C. Tardif, Fractional chromatic numbers of cones over graphs, J. Graph Theory, 38 (2001), 87-94.

[17] C. Tardif, personal communication, 2020.

[18] C. Tardif and D. Wehlau, Chromatic numbers of products of graphs: the directed and undirected versions of the Poljak-Rödl function, J. Graph Theory 51(2006), 33-36.

[19] C. Tardif and M. Wrochna, Hedetniemi's Conjecture and Strongly Multiplicative Graphs SIAM J. Discrete Math., 33(4)(2019), 22182250.

[20] C. Tardif and X. Zhu, A note on Hedetniemis conjecture, Stahlsconjecture and the Poljak-Rödl function, The electronic journal of combinatorics 26(4)(2019), \#P4.321

[21] M. Wrochna, Square-free graphs are multiplicative, Journal of Combinatorial Theory, Series B Volume 122, January 2017, Pages 479-507.

[22] X. Zhu, A survey on Hedetniemi's conjecture, Taiwanese J. Math. 2 (1998) 1-24.

[23] X. Zhu, The fractional version of Hedetniemi's conjecture is true, European J. Combin. 32 (2011), 1168-1175.

[24] X. Zhu, A note on the Poljak-Rödl function, The electronic journal of combinatorics (2020), to appear, arXiv:1911.12015. 